\title{
The impact of mental illness on potentially preventable hospitalisations: a population-based cohort study
}

\author{
Qun Mai ${ }^{1 *}$, C D'Arcy J Holman ${ }^{1}$, Frank M Sanfilippo ${ }^{1}$ and Jonathan D Emery ${ }^{2}$
}

\begin{abstract}
Background: Emerging evidence indicates an association between mental illness and poor quality of physical health care. To test this, we compared mental health clients (MHCs) with non-MHCs on potentially preventable hospitalisations (PPHs) as an indicator of the quality of primary care received.

Methods: Population-based retrospective cohort study of 139,208 MHCs and 294,180 matched non-MHCs in Western Australia from 1990 to 2006, using linked data from electoral roll registrations, mental health registry (MHR) records, hospital inpatient discharges and deaths. We used the electoral roll data as the sampling frame for both cohorts to enhance internal validity of the study, and the MHR to separate MHCs from non-MHCs. Rates of PPHs (overall and by PPH category and medical condition) were compared between MHCs, category of mental disorders and non-MHCs. Multivariate negative binomial regression analyses adjusted for socio-demographic factors, case mix and the year at the start of follow up due to dynamic nature of study cohorts.

Results: PPHs accounted for more than 10\% of all hospital admissions in MHCs, with diabetes and its complications, adverse drug events (ADEs), chronic obstructive pulmonary disease (COPD), convulsions and epilepsy, and congestive heart failure being the most common causes. Compared with non-MHCs, MHCs with any mental disorders were more likely to experience a PPH than non-MHCs (overall adjusted rate ratio (ARR) 2.06, 95\% confidence interval (CI) 2.03-2.09). ARRs of PPHs were highest for convulsions and epilepsy, nutritional deficiencies, COPD and ADEs. The ARR of a PPH was highest in MHCs with alcohol/drug disorders, affective psychoses, other psychoses and schizophrenia.

Conclusions: MHCs have a significantly higher rate of PPHs than non-MHCs. Improving primary and secondary prevention is warranted in MHCs, especially at the primary care level, despite there may be different thresholds for admission in people with established physical disease that is influenced by whether or not they have comorbid mental illness.
\end{abstract}

\section{Background}

Health care disparities in vulnerable populations are a public health and ethical challenge [1]. Previous studies have been predominately focused on racial/ethnic [2], socioeconomic [3] or geographic related disparities [4]. Mental illness related disparities have been given less attention [5].

About 1 in 5 Australian adults has a clinically diagnosable mental illness [6]. This vulnerable group not only

\footnotetext{
* Correspondence: qmai@meddent.uwa.edu.au

'School of Population Health, The University of Western Australia, 35 Stirling Highway, Crawley, WA, 6009, Australia

Full list of author information is available at the end of the article
}

suffer from debilitating disability and a high risk of suicide [7], but also high risks of morbidity and mortality from physical illness [8]. The 2000 Duty to Care study found that Western Australian (WA) mental health clients (MHCs), generally with moderate to severe mental illness, had an overall 2.5 times higher mortality rate than the general population, mostly due to preventable physical diseases [8]. Apart from genetic, lifestyle, social and environmental factors, disparities in access to, and the quality of, physical health care may also contribute to this [9]. Access to care is a prerequisite for quality of care, whilst primary care is a foundation for population health, especially for vulnerable groups [10]. Our

C Biomed Central

(c) 2011 Mai et al; licensee BioMed Central Ltd. This is an Open Access article distributed under the terms of the Creative Commons Attribution License (http://creativecommons.org/licenses/by/2.0), which permits unrestricted use, distribution, and reproduction in any medium, provided the original work is properly cited. 
previous study found that MHCs had substantially more general practitioner (GP) visits than non-MHCs [11]. This suggests that, in Australia, with its universal health insurance cover provided by Medicare, it appears unlikely that limited access to primary care explains poor physical health outcomes in MHCs. We have therefore turned our focus on whether disparities exist in the quality of primary care using potentially preventable hospitalisations (PPHs) as an indicator [12]. This is because the data we have cannot measure quality of primary care directly but indicators of it. These indicators of quality of primary care are useful screening tools for potential problems in preventive and primary care, and that determining whether there is a quality problem requires more in-depth analysis.

PPH medical conditions, also known as avoidable hospitalisation conditions or ambulatory care sensitive conditions, are those for which good primary and preventive care are thought that could potentially prevent the need for hospitalisation, and are thus considered as indicators for access to, and the quality of, primary care [12]. Early studies categorised PPH medical conditions into: vaccine-preventable, chronic and acute [13]. Adverse events are now also included, adding a safety measure of quality [13].

Previous studies have examined: (i) mental illnessrelated disparities in the quality of physical health care across several physical conditions, such as coronary heart disease [14] and diabetes mellitus [15]; and (ii) racial/ethnic, socioeconomic and geographic-related disparities in PPHs [16-18]. However, to our knowledge, no study has specifically examined mental illness-related disparities in PPHs. To address this, we linked multiple population-based datasets to answer four specific questions: (i) do MHCs have more PPHs than non-MHCs; (ii) which $\mathrm{PPH}$ category/medical condition has the highest relative risk; (iii) do the associations vary by category of mental disorders; and (iv) what would be the potential savings in hospital admissions if MHCs had received the 'same' quality of primary care as non-MHCs.

\section{Methods}

We conducted a population-based retrospective cohort study for the period 1 January 1990 to 30 June 2006 in WA.

\section{Data sources}

We linked four de-identified routinely collected datasets from the WA Data Linkage System [19,20] (see Additional File 1, Table S1): (i) mental health registry (MHR, $8 \%$ of the general population), (ii) electoral roll registrations ( $86 \%$ of the general population aged $\geq 18$ years), (iii) hospital inpatient discharges, and (iv) death registrations.
The MHR contained mental health inpatient data from all psychiatric institutions, public and private general hospitals, and outpatient data from public mental health clinics, community mental health services and psychiatric residential units. Data from private psychiatrists and GPs treating mental disorders were collected and administered by the Commonwealth Department of Health and thus not covered in the MHR.

\section{Study cohorts}

To enhance the internal validity of the study, we used the electoral roll as the sampling frame for both MHCs and non-MHCs to ensure that the baseline populations - MHCs and non-MHCs - came ultimately from the same source (Figure 1). The WA state-wide electoral roll data is a single dataset that contains the complete set of 1988 WA state electoral roll registrations and all quarterly updates of changes of enrolment status since then, e.g. new enrolments as well as removals from the roll due to unsound mind, moved out of state or death. The dataset contains information on encrypted ID, date of transaction, reason for the transaction, sex, date of birth and place of residence. MHCs were defined as people on the electoral roll, who were also on the MHR (about 80\% of MHR) and still alive from 1 January 1990 onwards. Non-MHCs were a random sample of people who were on the electoral roll, but never recorded in the MHR. They were matched 2:1 with MHCs by 5-year age group, sex and current electoral roll registration at study entry. Age is calculated from date of birth and study entry date. For MHCs, the study entry date was 1 January 1990 for patients recorded in the MHR before 1 January 1990, or the first date of registration on the MHR for those recorded later. For non-MHCs, it was the same as that of their matched MHCs. The start of follow-up $\left(\mathrm{T}_{0}\right)$ was the entry date for both cohorts.

\section{Variables and measurements Outcome variables}

The outcome measure was the rate of PPHs during the follow-up period from 1 Jan 1990 to 31 Dec 2006. There were four categories of PPH medical conditions investigated (see Additional File 1, Table S2): vaccinepreventable, chronic, acute and adverse drug events (ADEs). The first three categories were identified using the Australian Institute of Health and Welfare definition [21], with diabetes-related renal dialysis being counted only once for each person. This was because there were a large number of diabetes-related renal dialysis admissions, and for the purpose of this study we considered these as one episode of care per person and counted as one hospital discharge with a length of stay of one day. We substituted adverse events with ADEs as the majority of advents events were ADEs, defined as any adverse 


\section{Western Australian Data Linkage System}

Including ER (from 1988), MHR (from 1966), hospital inpatient (from 1990), death data (from 1990)

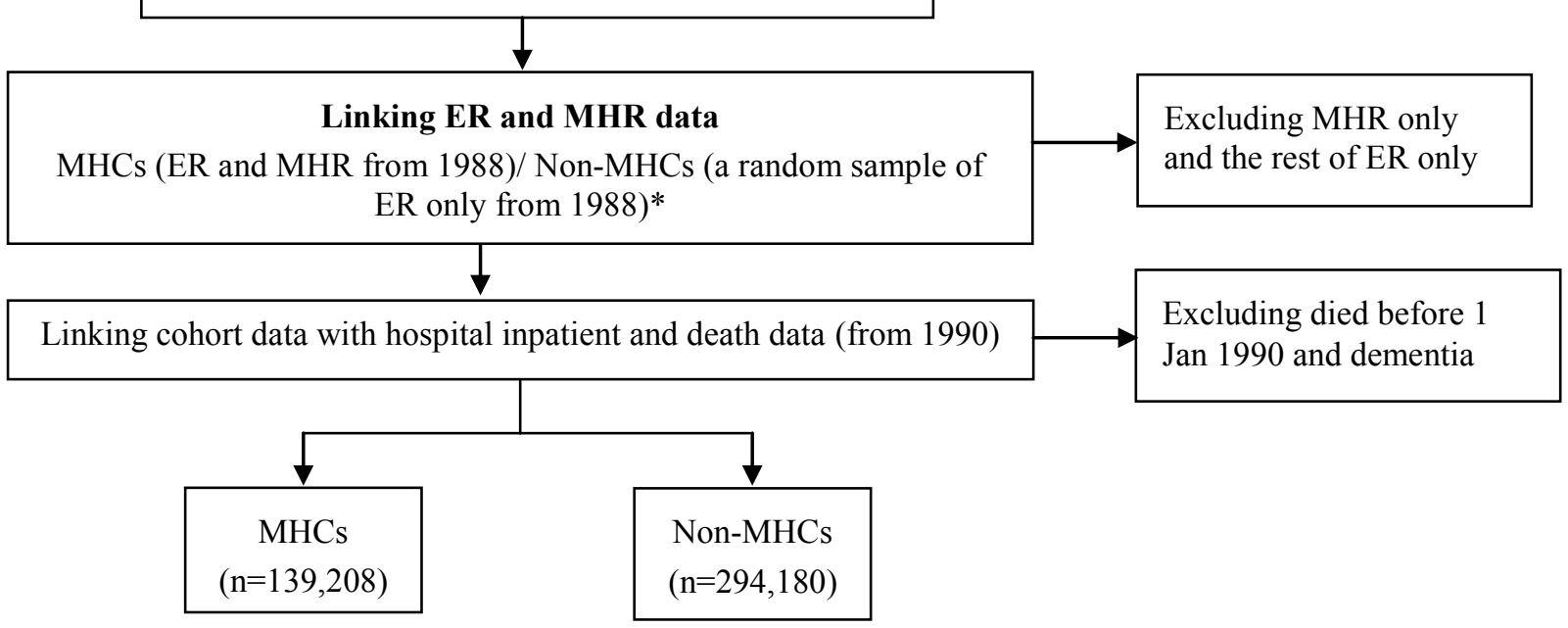

Figure 1 Selection of study cohorts. Abbreviations: $E R=$ electoral roll registrations, MHCs = mental health clients, MHR = mental health registry. ${ }^{*}$ Non-MHCs matched 2:1 with MHCs by 5-year age group, sex and being a current elector at study entry. The final ratio of non-MHC to MHC was 2.11:1 after excluding MHCs with dementia.

effect of drugs, medicines or biological substances in therapeutic use (ICD-9-CM, E930-E949 or ICD-10-AM, Y40-Y59). Follow-up time was censored at 30 June 2006 or an earlier date of death.

\section{Exposure variables}

We ascertained the principal mental health diagnosis for each MHC using a previously published method [11,22], which assigned patients to their most significant mental health diagnosis on an hierarchy of severity (see Additional File 1, Table S1). People with dementia ( $\mathrm{n}=$ $15,764)$ were excluded due to their high use of residential care, but their matched non-MHCs were retained to maintain the high precision possible in a study of this size. The remaining records were then grouped into one of ten mutually exclusive categories of mental disorders (see Additional File 1, Table S1).

\section{Potential confounders}

Scores for social disadvantage and residential remoteness were derived from the Index of Relative Socio-Economic Disadvantage (IRSD) [23] and the Accessibility/ Remoteness Index of Australia (ARIA) [24] based on place of residence at the Australian Census date. Social disadvantage scores were grouped into five levels (the lowest $10 \%$ of IRSD scores of the WA general population, $10 \%$ to $<25 \%, 25 \%$ to $<50 \%, 50 \%$ to $<75 \%$ and $\geq$ $75 \%)$ and remoteness scores were grouped into metropolitan, rural and remote. The category of the lowest $10 \%$ IRSD was created because a high proportion of MHCs fell into this group. Age, level of social disadvantage, level of residential remoteness and year at the start of follow up were measured at $\mathrm{T}_{0}$. Physical comorbidities were measured by the Charlson Index [25] based on inpatient data with a five-year look-back period from $\mathrm{T}_{0}$.

\section{Statistical analysis}

We compared patient characteristics and crude numbers of hospital discharges, bed days and average length of stay during the entire follow-up period between MHCs and non-MHCs using bivariate analyses (chi-squared or unpaired tests for categorical variables, two-tailed $t$ - or Mann-Whitney tests for continuous variables).

We then compared rates of PPHs between the two cohorts using unadjusted and adjusted negative binomial regression. Adjusted analyses controlled for potential patient-level confounders: 5-year age group, sex, Indigenous status, level of social disadvantage, level of residential remoteness, physical comorbidities (Charlson Index as a continuous variable) and year at $\mathrm{T}_{0}$. We repeated the above analyses for each $\mathrm{PPH}$ category and condition. Because MHCs represented a heterogeneous group, we repeated the above analyses, comparing $\mathrm{MHCs}$ in each category of mental disorder with non$\mathrm{MHCs}$.

We calculated the etiological fraction of mental illness attributable to disparities in PPHs as (Rate Ratio-1)/Rate Ratio and estimated the potential savings in hospital admissions and bed days if MHCs had experienced the 
same PPH outcomes as non-MHCs, determined by multiplying hospital discharges by the etiological fraction.

Missing values for each variable were treated as a separate exposure category so that all subjects were included in the multivariate analyses. Stata version 10.0 for Windows (StataCorp, College Station, Tex, USA) was used for all analyses.

The study was approved by the Human Research Ethics Committees of The University of Western Australia, and health departments of the Australian and WA governments.

\section{Results}

Patient characteristics

The study cohorts comprised 139,208 MHCs and 294,180 non-MHCs. Characteristics of MHCs and nonMHCs at $\mathrm{T}_{0}$ are shown in Table 1. Relative to nonMHCs, MHCs were more likely to be Indigenous, socially disadvantaged, living in rural or remote WA and have more physical comorbidities (all p-values $<0.001$ ). The distribution of mental disorders among MHCs is also shown in Table 1, with affective psychoses and neurotic disorders being the most common.

\section{Descriptive analyses}

Numbers of hospital discharges, bed days and average length of stay of total hospital admissions and PPHs (total and by PPH category and medical condition) for both cohorts during entire follow-up period are shown in Table 2. PPHs accounted for more than $10 \%$ of all hospital discharges in both groups (Table 2). The most common PPH medical conditions in MHCs were diabetes and its complications, ADEs, chronic obstructive pulmonary disease (COPD), convulsions and epilepsy, and congestive heart failure. ADEs, diabetes and its complications, COPD and congestive heart failure had the highest numbers of total bed days. PPH medical conditions with the longest average length of stay were

Table 1 Characteristics of mental health clients (MHCs) and non-MHCs at the start of follow up

\begin{tabular}{|c|c|c|}
\hline Characteristic* & $\begin{array}{c}\text { MHCs } \\
(\mathrm{n}=139,208)\end{array}$ & $\begin{array}{c}\text { Non-MHC } \\
(n=294,180)\end{array}$ \\
\hline Age, years, mean (SD) & $43.7(18.6)$ & $45.1(19.7)$ \\
\hline Sex, $\%$ male & $40.3 \%$ & $40.4 \%$ \\
\hline \multicolumn{3}{|l|}{ Indigenous status, $\%$} \\
\hline Indigenous (excluding missing) & $5.7 \%(5.7 \%)$ & $2.1 \%(2.3 \%)$ \\
\hline Non-Indigenous (excluding missing) & $93.9 \%(94.3 \%)$ & $87.9 \%(97.7 \%)$ \\
\hline Missing & $0.4 \%$ & $10.0 \%$ \\
\hline \multicolumn{3}{|l|}{ Level of social disadvantage } \\
\hline Most disadvantaged (the lowest $10 \%$ of IRSD scores**) & $14.8 \%$ & $10.9 \%$ \\
\hline More disadvantaged ( $10 \%$ to $<25 \%)$ & $18.7 \%$ & $15.8 \%$ \\
\hline Little disadvantaged ( $25 \%$ to < $50 \%$ ) & $25.4 \%$ & $23.6 \%$ \\
\hline Less disadvantaged ( $50 \%$ to < $75 \%$ ) & $18.5 \%$ & $20.1 \%$ \\
\hline Least disadvantaged $(75 \%+)$ & $22.6 \%$ & $29.6 \%$ \\
\hline \multicolumn{3}{|l|}{ Residential remoteness } \\
\hline Metropolitan WA & $68.6 \%$ & $73.0 \%$ \\
\hline Rural WA & $22.7 \%$ & $20.6 \%$ \\
\hline Remote WA & $8.7 \%$ & $6.4 \%$ \\
\hline Physical comorbidity (Charlson) score, mean (SD) & $1.28(2.34)$ & $1.04(2.24)$ \\
\hline \multicolumn{3}{|l|}{ Category of mental disorders (\%) } \\
\hline Alcohol/drug disorders & $8.0 \%$ & - \\
\hline Schizophrenia & $4.2 \%$ & - \\
\hline Affective psychoses & $17.1 \%$ & - \\
\hline Other psychoses & $6.7 \%$ & - \\
\hline Neurotic disorders & $16.2 \%$ & - \\
\hline Personality disorders & $2.5 \%$ & - \\
\hline Adjustment disorders & $7.3 \%$ & - \\
\hline Depressive disorders & $4.4 \%$ & - \\
\hline Other mental disorders & $8.0 \%$ & - \\
\hline In MHR but had no mental health diagnosis, including suicide attempts & $25.5 \%$ & - \\
\hline
\end{tabular}

Abbreviations: $\mathrm{MHCs}=$ mental health clients, $\mathrm{SD}=$ standard deviation, WA = Western Australia, MHR = mental health registry.

*P-values $<0.001$ for all comparisons between MHCs and non-MHCs, except for sex $(p=0.52)$.

**The lowest $10 \%$ of IRSD scores of the Western Australian general population. 
Table 2 Hospital discharges, bed days and average length of stay for potentially preventable hospitalisations by study cohort, PPH category and condition, 1 January 1990 to 30 June 2006

\begin{tabular}{|c|c|c|c|c|c|c|}
\hline PPH category/condition & $\begin{array}{c}\text { Discharges in } \\
\text { MHCs } \\
(n=139,208)\end{array}$ & $\begin{array}{c}\text { Discharges in } \\
\text { Non-MHC } \\
(n=294,180)\end{array}$ & $\begin{array}{c}\text { Bed-days in } \\
\text { MHCs } \\
\text { (n = 139,208) }\end{array}$ & $\begin{array}{c}\text { Bed-days in } \\
\text { Non-MHC } \\
(n=294,180)\end{array}$ & $\begin{array}{c}\text { ALOS (days) in } \\
\text { MHCs } \\
(n=139,208)\end{array}$ & $\begin{array}{c}\text { ALOS (days) in } \\
\text { Non-MHC } \\
(n=294,180)\end{array}$ \\
\hline Vaccine-preventable* & 3,055 & 3,205 & 38,465 & 35,819 & 12.6 & 11.2 \\
\hline Influenza and pneumonia & 2,786 & 2,879 & 35,847 & 33,154 & 12.9 & 11.5 \\
\hline Other vaccine-preventable conditions & 270 & 328 & 2,686 & 2,682 & 9.9 & 8.2 \\
\hline Chronic $^{*}$ & 48,350 & 62,644 & 309,642 & 403,664 & 6.4 & 6.4 \\
\hline Asthma & 6,483 & 4,337 & 31,435 & 21,496 & 4.8 & 5.0 \\
\hline Congestive heart failure & 6,844 & 13,138 & 58,954 & 121,571 & 8.6 & 9.3 \\
\hline Diabetes complications† & 17,823 & 23,837 & 115,418 & 149,139 & 6.5 & 6.3 \\
\hline COPD & 9,703 & 10,466 & 87,385 & 93,766 & 9.0 & 9.0 \\
\hline Angina & 6,292 & 8,680 & 19,157 & 28,029 & 3.0 & 3.2 \\
\hline Iron deficiency anaemia & 2,466 & 4,469 & 5,936 & 10,503 & 2.4 & 2.4 \\
\hline Hypertension & 1,497 & 1,598 & 7,731 & 7,214 & 5.2 & 4.5 \\
\hline Nutritional deficiencies & 21 & 10 & 363 & 289 & 17.3 & 28.9 \\
\hline Rheumatic heart disease & 304 & 521 & 2,519 & 4,262 & 8.3 & 8.2 \\
\hline Acute $^{*}$ & 33,329 & 30,720 & 148,426 & 156,055 & 4.5 & 5.1 \\
\hline Dehydration and gastroenteritis & 5,578 & 6,404 & 17,696 & 20,861 & 3.2 & 3.3 \\
\hline Pyelonephritis & 6,005 & 6,408 & 35,220 & 40,422 & 5.9 & 6.3 \\
\hline Perforated/bleeding ulcer & 1,090 & 1,737 & 8,000 & 13,040 & 7.3 & 7.5 \\
\hline Cellulitis & 4,334 & 4,574 & 23,089 & 28,398 & 5.3 & 6.2 \\
\hline Pelvic inflammatory disease & 1,622 & 1,366 & 4,922 & 3,908 & 3.0 & 2.9 \\
\hline Ear, nose and throat infections & 1,989 & 1,810 & 6,460 & 6,038 & 3.2 & 3.3 \\
\hline Dental conditions & 3,724 & 5,147 & 5,812 & 6,658 & 1.6 & 1.3 \\
\hline Appendicitis with generalised peritonitis & 244 & 497 & 2,096 & 3,138 & 8.6 & 6.3 \\
\hline Convulsions and epilepsy & 8,036 & 1,830 & 31,376 & 9,259 & 3.9 & 5.1 \\
\hline Gangrene & 726 & 980 & 14,050 & 24,460 & 19.4 & 25.0 \\
\hline Adverse drug events & 16,002 & 15,683 & 211,495 & 174,786 & 13.2 & 11.1 \\
\hline Total PPHs* & 96,862 & 107,821 & 665,517 & 720,975 & 6.9 & 6.7 \\
\hline Total hospital admissions $\dagger$ & 912,175 & $1,013,403$ & $5,579,134$ & $4,413,672$ & 6.1 & 4.4 \\
\hline
\end{tabular}

Abbreviations: $\mathrm{MHCs}=$ mental health clients, $\mathrm{PPH}=$ potentially preventable hospitalisations, $\mathrm{COPD}=$ chronic obstructive pulmonary disease, $\mathrm{ALOS}=$ average length of stay (days).

*There were overlaps in definitions for PPHs, therefore the total numbers for each PPH categories and total PPHs were not the same as the sum of individual PPHs. †During follow-up period, there were a total of 183,846 hospital separations for renal dialysis among 919 persons. For the purpose of this study, all renal dialysis admissions were counted as one hospital episode for each person with length of stay of one day. The same definition was also applied for diabetesrelated renal dialysis.

gangrene, nutritional deficiencies, ADEs, and influenza and pneumonia.

\section{Rate of PPHs}

Compared with non-MHCs, MHCs had significantly higher rates of PPHs from both univariate and multivariate analyses (unadjusted rate ratio (RR) 2.12, 95\% CI 2.07-2.16; adjusted RR (ARR) 2.06, 2.03-2.09) (Figure 2).

\section{By PPH category and condition}

When we stratified analyses by PPH category and specific condition, ARRs were greater than one for all PPH categories and medical conditions, except appendicitis with generalised peritonitis $(0.88,0.75-1.03)$ (Table 3$)$. By PPH category, ARRs were highest for ADEs (2.66, 2.58-2.74), followed by vaccine-preventable $(2.10,1.97-2.23)$, acute
(2.08, 2.04-2.13) and chronic conditions (1.82, 1.78-1.87). By PPH medical conditions, ARRs were highest for convulsions and epilepsy $(6.45,5.89-7.07)$, nutritional deficiencies $(4.81,1.43-16.21)$, COPD $(2.64,2.47-2.83)$ and asthma (2.47, 2.30-2.66); although nutritional deficiencies had the lowest absolute numbers (21 in MHCs and 10 in non-MHCs). Other findings included that ARR increased with the year at the start of follow up (ARR 1.02, 95\% 1.01-1.02, for each increment in year), age, social disadvantage, residential remoteness and level of physical comorbidities. It was also greater in females than males and Indigenous people than non-Indigenous people.

Rate ratios by category of mental disorders

ARRs of PPHs in MHCs with any mental disorders were all greater than one (Figure 3), with the highest for 


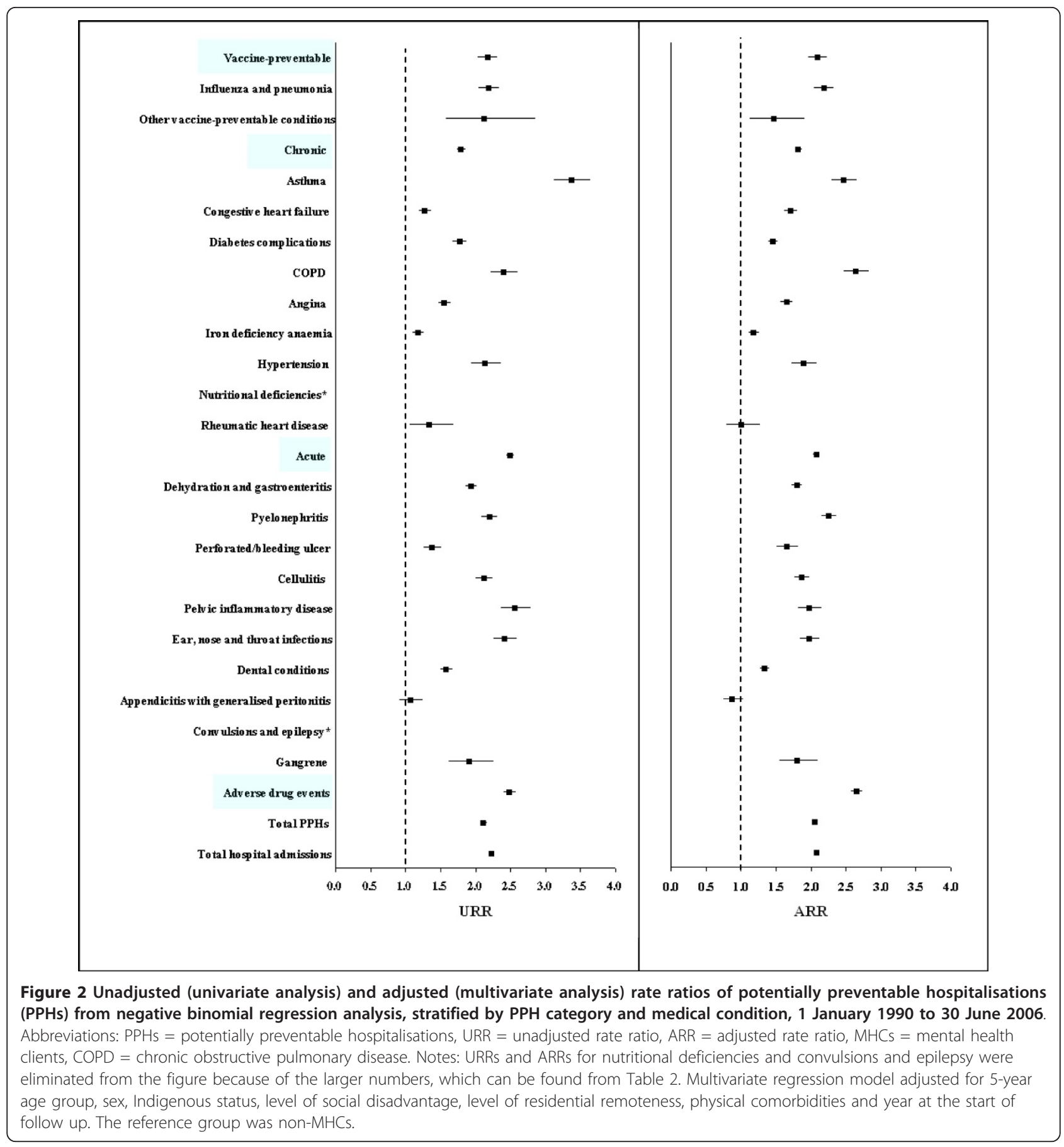

alcohol and drug disorders (3.00, 2.88-3.13), affective disorders $(2.58,2.50-2.66)$, other psychoses $(2.36,2.26$ $2.47)$ and schizophrenia $(2.25,2.12-2.39)$. The leading causes of excess PPHs for these four categories of mental disorders are shown in Table 4.

\section{Potential savings}

Greenland and Robins have described three different types of attributable fraction in the exposed [26]. In this study, the incidence density fraction of PPHs in MHCs attributable to their mental illness was $51.4 \%$, based on an ARR of 2.06 (Table 3). Strictly, the incidence density fraction will only equal the true etiologic fraction when exposure acts independently of background causes [27], but otherwise the approximation is often conservative [28]. Thus, a rough estimate of the potential savings is that if the elevated rate of PPHs in the mentally ill observed in Western Australia was caused by 
Table 3 Unadjusted (univariate analysis) and adjusted (multivariate analysis) rate ratios of potentially preventable hospitalisations (PPHs) from negative binomial regression analysis, stratified by PPH category and medical condition, 1 January 1990 to 30 June 2006

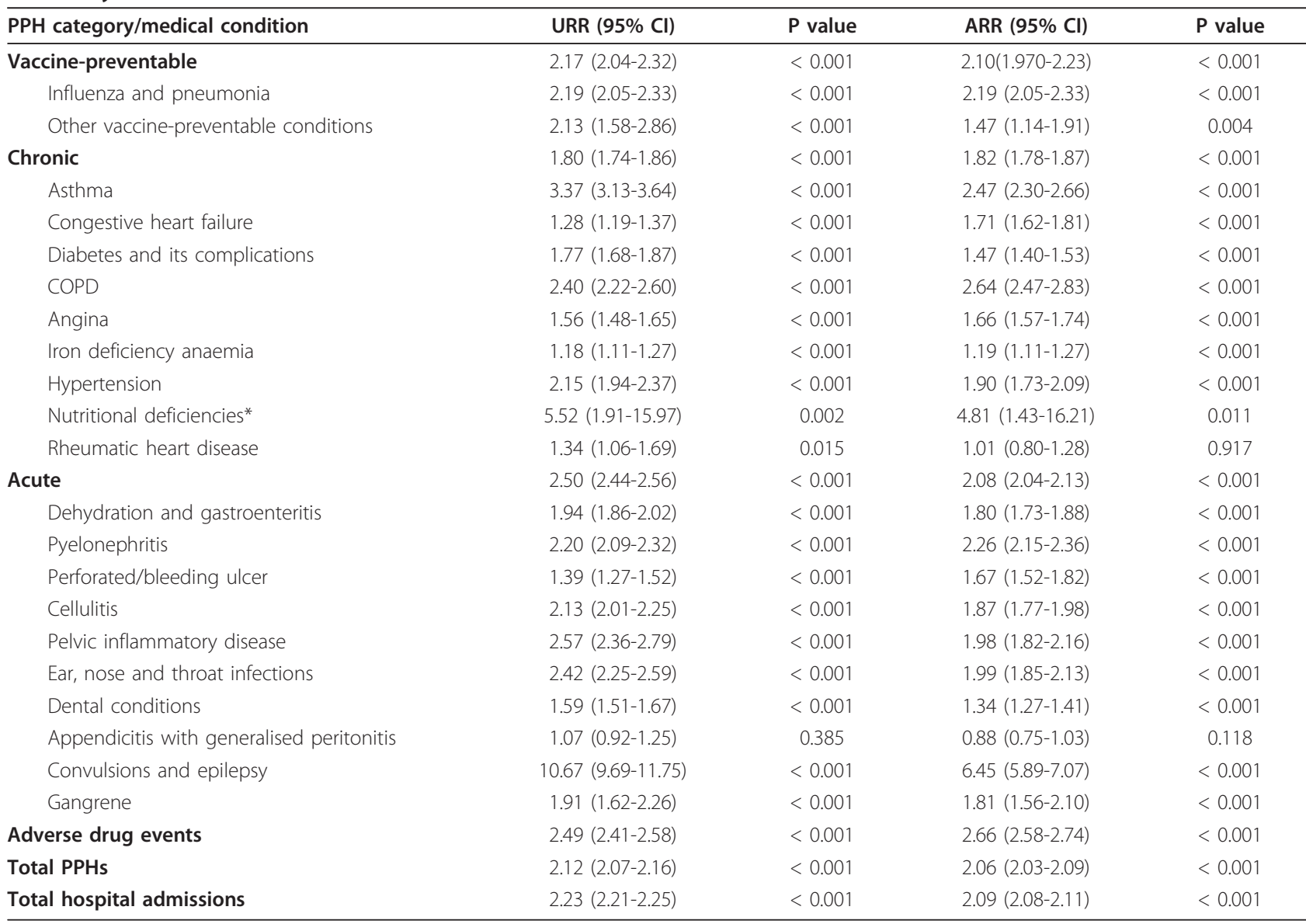

Abbreviations: $\mathrm{PPH}$ = potentially preventable hospitalisations, $\mathrm{URR}=$ unadjusted rate ratio, $\mathrm{ARR}=$ adjusted rate ratio, $\mathrm{COPD}=\mathrm{chronic}$ obstructive pulmonary disease.

Unadjusted and adjusted rate ratios of hospitalisation were the second highest for nutritional deficiencies, after convulsions and epilepsy, but there were only 21 cases in MHCs and 10 in non-MHCs during the entire follow up period. Multivariate regression model adjusted for 5-year age group, sex, Indigenous status, level of social disadvantage, level of residential remoteness, physical comorbidities and year at the start of follow up.

The reference group was the comparison cohort, non-MHCs.

suboptimal health care, then had that inequality in care been redressed, it could have translated into 49,787 (i.e., $96,862 \times 51.4 \%)$ fewer hospital admissions at the times that they occurred during follow-up.

\section{Discussion}

We used a population-based approach to examine mental illness related disparities in PPHs. The results showed that: (i) diabetes and its complications, ADEs, COPD, convulsions and epilepsy, and congestive heart failure were the most common PPHs in MHCs; (ii) on average, MHCs were about twice as likely as non-MHCs to experience a $\mathrm{PPH}$, with the largest differences occurring in PPHs for convulsions and epilepsy, nutritional deficiencies, ADEs, COPD and asthma; (iii) although ARRs were greater than 1 in MHCs with any mental disorders, it was higher in those with relatively more debilitating mental disorders such as alcohol and drug disorders, affective psychoses, other psychoses and schizophrenia; (iv) the disparities seem to have been increasing over the years; and (v) potentially half of all acute hospital admissions for PPHs could be avoided if MHCs had received preventive and primary care that achieved the same PPH outcomes as observed in non-MHCs.

The strengths of our study were: (i) use of validated population-based linked data with over 400,000 people in the study populations, (ii) inclusion of wide spectrums of mental disorders and PPH medical conditions, (iii) use of an internally valid comparison cohort of nonMHCs, and (iv) long-term follow-up (up to 16.5 years). It allows the scope of the problem to be quantified using innovative methods from well-established datasets with complete capture of population-wide data, so that changes in the situation can be monitored and the 


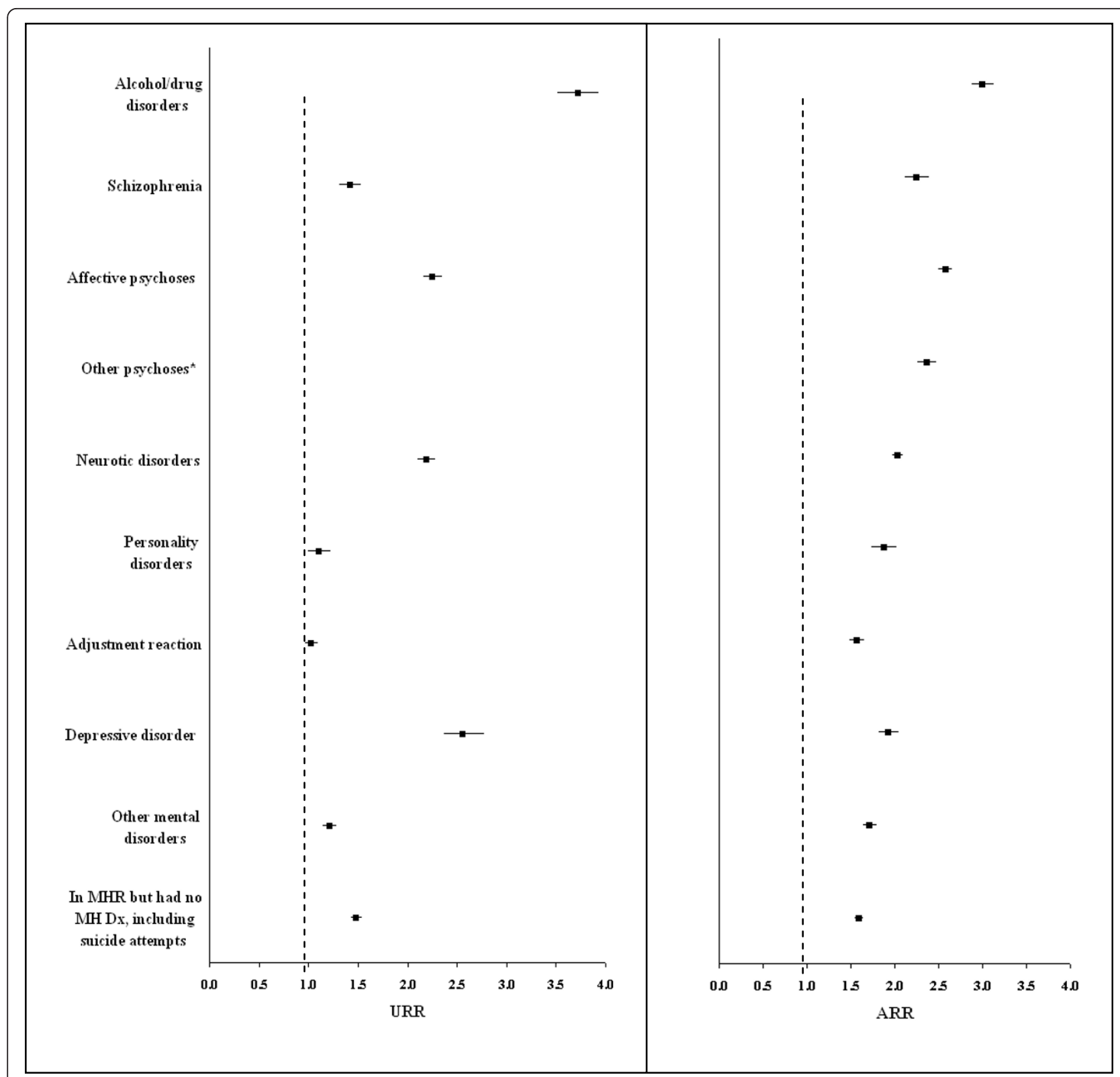

Figure 3 Unadjusted (univariate analysis) and adjusted (multivariate analysis) rate ratios of total potentially preventable hospitalisations (PPHs) from negative binomial regression analysis, stratified by category of mental disorders. Abbreviations: MHR $=$ mental health registry, $\mathrm{MH}=$ mental health, $\mathrm{Dx}=$ diagnosis, $\mathrm{URR}=$ unadjusted rate ratio, ARR = adjusted rate ratio, MHCs = mental health clients. Note: URR for other psychoses was eliminated from the figure because of the large number (URR 6.42, 95\% Cl 6.02-6.85). The drop in other psychoses from an URR of 6.42 to an ARR of 2.47, mainly due to their older age and high level of physical comorbidities. Multivariate regression model adjusted for 5-year age group, sex, Indigenous status, level of social disadvantage, level of residential remoteness, physical comorbidities and year at the start of follow up. The reference group was non-MHCs.

effectiveness of large-scale interventions and policy changes can be examined.

Limitations included firstly we did not have the data to directly measure the quality of preventive/primary care but an indicator of it. Thus, we cannot answer the question about whether higher rates of PPHs are due to poor quality of primary care or other factors.
Nevertheless, the results show that there may be potential problems in preventive/primary care in MHCs that warrant more in-depth analysis. Secondly, the lack of data on ambulatory services provided by private psychiatrists and GPs treating mental disorders. This limited the extrapolation of our findings for all people with mental illness because some people with mental illness 
Table 4 Adjusted rate ratios of potentially preventable hospitalisations, by selective categories of mental disorders

\begin{tabular}{|c|c|c|}
\hline Category of mental disorder and $\mathrm{PPH}$ & ARR & $95 \% \mathrm{Cl}$ \\
\hline \multicolumn{3}{|l|}{ Alcohol and drug disorders } \\
\hline Nutritional deficiencies & 19.45 & $(1.79-211.88)$ \\
\hline Convulsions and epilepsy & 16.35 & $(13.47-19.84)$ \\
\hline Gangrene & 5.22 & $(3.86-7.05)$ \\
\hline COPD & 4.49 & $(2.57-7.85)$ \\
\hline Other vaccine-preventable conditions & 4.47 & $(3.76-5.30)$ \\
\hline Perforated/bleeding ulcer & 4.21 & $(3.46-5.11)$ \\
\hline \multicolumn{3}{|l|}{ Affective disorders } \\
\hline Nutritional deficiencies & 18.68 & $(2.49-140.27)$ \\
\hline Convulsions and epilepsy & 6.51 & $(5.59-7.59)$ \\
\hline Adverse drug events & 4.53 & $(4.31-4.78)$ \\
\hline Asthma & 2.98 & $(2.59-3.41)$ \\
\hline COPD & 2.98 & $(2.59-3.40)$ \\
\hline \multicolumn{3}{|l|}{ Other psychoses } \\
\hline Convulsions and epilepsy & 19.63 & $(15.94-24.17)$ \\
\hline Pyelonephritis & 3.22 & $(2.89-3.58)$ \\
\hline Adverse drug events & 3.11 & $(2.89-3.36)$ \\
\hline Other vaccine-preventable conditions & 2.91 & $(1.33-6.38)$ \\
\hline Gangrene & 2.50 & $(1.78-3.51)$ \\
\hline \multicolumn{3}{|l|}{ Schizophrenia } \\
\hline Nutritional deficiencies & 53.48 & $(1.97-1451.32)$ \\
\hline Adverse drug events & 6.91 & $(6.30-7.58)$ \\
\hline Convulsions and epilepsy & 5.97 & $(4.52-7.87)$ \\
\hline Congestive heart failure & 2.91 & $(2.34-3.62)$ \\
\hline Influenza and pneumonia & 2.63 & $(2.11-3.27)$ \\
\hline
\end{tabular}

Abbreviations: $\mathrm{ARR}=$ adjusted rate ratio, $\mathrm{COPD}=$ chronic obstructive pulmonary disease.

in Australia receive treatment only through these private sectors. Nevertheless, the MHR included about $40 \%$ of people with mental illness, generally with moderate to severe illness, whose physical health and physical health care disparities were probably greater than the remainder of people with mental illness. Moreover, we used the MHR for selection of our mental health cohort as did the previous Duty to Care study [8] and GP utilisation study [11], thus ensuring continuity and integrity of our investigations and findings. Thirdly, the domain restriction to the electoral roll, which enhanced the internal validity, possibly reduced external validity. Disparities may be greater in MHCs who are not registered to vote (20\% of the MHR), presumably those younger than 18 years old, with severe mental illness, homeless and new migrants. Moreover, the MHR captured only $40 \%$ of patients with mental illness, thus our non-MHCs almost certainly included some people with mental illness. This may have resulted in an underestimation of the true difference between MHCs and non-MHCs. Fourthly, the lack of information on lifestyle risk factors (e.g., smoking and obesity) or detailed clinical information (e.g., severity of disease) limited our adjustment for these factors in the analyses.

The significance and interpretation of the study findings need to take into account both absolute and relative measures. PPHs with both higher absolute numbers and ARRs deserve special attention, such as, diabetes and its complications, ADEs, COPD, and convulsions and epilepsy. Compared with non-MHCs, MHCs are more likely to have a higher prevalence of underlying PPH medical conditions $[29,30]$ therefore have higher risks of hospitalisations for $\mathrm{PPH}$ medical conditions. Adequate access to and good quality of preventive and primary care are thought to lower the risks of hospitalisations for PPH medical conditions [12]. Our previous study reported that MHCs visit GPs significantly more often than non-MHCs, suggesting that the differences in the quality of primary care rather than access to primary care may deserve further investigations.

Although access to ambulatory specialist care may also impact on the risk of PPHs, primary care, not specialist care, is the ideal setting for primary and secondary prevention of $\mathrm{PPH}$ medical conditions, especially in MHCs with multiple comorbidities [31]. This is attributable to the core features of primary care: first point of contact, continuity, comprehensiveness, coordination and its lower cost [10].

The greatest disparities in patients with alcohol and drug disorders warrant special attention, as other work suggests that they are unlikely to receive preventive care [32]. Studies on race-related health care disparities have suggested that patient-provider interactions may be a major contributor to the disparities, thus the interpersonal aspects of the patient-provider relationship may contribute to more pronounced disparities in patients with alcohol and drug disorders [5].

Schizophrenia and affective psychoses are severe mental disorders. These disorders are associated with a high prevalence of lifestyle risk factors (eg. smoking and obesity), comorbid physical diseases and alcohol and drug disorders, poly-pharmacy and their adverse effects [30]. These, together with functional disabilities of patients who may be under the care of multiple health care professionals, increase risks of PPHs, especially for diabetes, ADEs and COPD.

The combination of high physical health needs and a poor quality of physical health care received has been suggested as the hallmark of medically vulnerable populations, including people with mental illness. Studying PPHs is a way to quantify the scope of the problem and the scope for health gain. Our study suggests that mental illness-related disparities in physical disease burden are real and substantial and poor quality of primary care may be a contributor. However, some apparent PPHs may be appropriate in those with mental illness because 
the threshold for admission may need to be lower if someone has a co-morbid mental condition which limits their functional ability.

The observation that differences between levels of healthcare according to mental health status is getting worse over time is interesting. This may be partly due to the combination of: (i) the dramatic deinstitutionalisation movement of the mental health reform that transforms mental health services from an institutionbased to community-based care model, and (ii) inadequate supportive services and funding for supporting this movement so that people with mental illness may be more likely to fall through the cracks.

Further research is needed to examine in-depth whether there is a quality problem in primary care and to understand the extent to which patient, provider and system factors contribute to the quality of primary care and its implications for the outcomes of care and interventions.

\section{Conclusions}

MHCs have a significantly higher risk of PPHs than nonMHCs. They deserve special attention in research, policy development and clinical practice, with the focus on improving primary and secondary prevention, especially at the primary care level. This is despite the different thresholds for admission in people with established physical disease that is influenced by whether or not they have comorbid mental illness, which is encouraging.

\section{Additional material}

Additional file 1: Table S1 - Data sources and definitions. Table S1 shows data sources used in this study and definitions for severity of mental illness and category of mental disorders. Table S2 - ICD codes used for identifying potentially preventable hospitalisations. Table S2 shows ICD codes used for identifying potentially preventable hospitalisations.

\footnotetext{
Abbreviations

ADE: Adverse Drug Events; ARIA: Accessibility/Remoteness Index of Australia; ARR: Adjusted Rate Ratio; Cl: Confidence Interval; COPD: Chronic Obstructive Pulmonary Disease; GP: General Practitioner; ICD-9-CM: The International Classification of Diseases; $9^{\text {th }}$ revision; Clinical Modification; ICD-10-AM: International Classification of Disease $-10^{\text {th }}$ revision - Australian Modification; IRSD: Index of Relative Socio-Economic Disadvantage; MHCs: Mental Health Clients; MHR: Mental Health Registry; PPHs: Potentially Preventable Hospitalisations; RR: Rate Ratio; $T_{0}=$ start of follow up; URR: Unadjusted Rate Ratio; WA: Western Australia.

\section{Acknowledgements}

We thank both the federal and Western Australian Departments of Health, and Medicare Australia, for providing the datasets for analysis. We also thank the Data Linkage Branch at the Western Australian Department of Health for selecting study cohorts, linking and extracting associated linked records. This study was supported by a National Health and Medical Research Council (NHMRC) grant on chronic disease management in primary care (support obtained by $(D J H)$.
}

\section{Author details}

${ }^{1}$ School of Population Health, The University of Western Australia, 35 Stirling Highway, Crawley, WA, 6009, Australia. ${ }^{2}$ School of Primary, Aboriginal and Rural Health Care, The University of Western Australia, 35 Stirling Highway, Crawley, WA, 6009, Australia.

\section{Authors' contributions}

$\mathrm{QM}$ and $\mathrm{CDJH}$ participated in the conception and design of the overall study, and formulation of analysis plan. QM researched data and wrote the manuscript. CDJH and FMS reviewed and edited the manuscript and contributed to the discussion. JDE critically revised the manuscript for important intellectual content. All authors have read and approved the final manuscript.

\section{Competing interests}

The authors declare that they have no competing interests.

Received: 16 April 2011 Accepted: 10 October 2011

Published: 10 October 2011

\section{References}

1. Agency for Healthcare Research and Quality: National healthcare disparities report Rockville, MD; 2003 [http://www.ahrq.gov/qual/nhdr03/fullreport/.]

2. Smedley B, Stith A, Nelson A: Unequal treatment: confronting racial and ethnic disparities in healthcare Washington, D.C. National Academies Press; 2003.

3. McNiece R, Majeed A: Socioeconomic differences in general practice consultation rates in patients aged 65 and over: prospective cohort study. BMJ 1999, 319:26-8.

4. Wennberg J, Gittelsohn : Small area variations in health care delivery. Science 1973, 182:1102-8.

5. Frayne SM, Halanych JH, Miller DR, Wang F, Lin H, Pogach $L$, et al: Disparities in diabetes care: impact of mental illness. Arch Intern Med 2005, 165:2631-8.

6. Australian Bureau of Statistics: National health survey: mental health, Australia, 2001 Cat. no. 4811.0 Canberra; 2003.

7. Murray $\mathrm{CL}$, Lopez AD: The global burden of disease: a comprehensive assessment of mortality and disability from diseases, injuries, and risk factors in 1990 and projected to 2020 Cambridge, MA: Harvard University; 1996.

8. Lawrence D, Holman CDJ, Jablensky AV: Preventable physical illness in people with mental illness Western Australia; 2001.

9. Maj M: Physical health care in persons with severe mental illness: a public health and ethical priority. World Psychiatry 2009, 8:1-2.

10. Starfield B, Shi L, Macinko J: Contribution of primary care to health systems and health. Milbank Q 2005, 83:457-502.

11. Mai Q, Holman CD, Sanfilippo FM, Emery JD, Stewart LM: Do users of mental health services lack access to general practitioner services? Med J Aust 2010, 192:501-6.

12. Agency for Healthcare Research and Quality: AHRQ quality indicators guide to prevention quality indicators: hospital admission for ambulatory care sensitive conditions. Version 3.1, Washington D.C.; 2007.

13. Clinical Epidemiology and Health Service Evaluation Unit: Potentially preventable hospitalisations: a review of the literature and Australian policies. 2009.

14. Hippisley-Cox J, Parker C, Coupland C, Vinogradova Y: Inequalities in the primary care of patients with coronary heart disease and serious mental health problems: a cross-sectional study. Heart 2007, 93:1256-62.

15. Jones LE, Clarke W, Carney CP: Receipt of diabetes services by insured adults with and without claims for mental disorders. Med Care 2004, 42:1167-75.

16. Ansari Z, Barbetti T, Carson NJ, Auckland MJ, Cicuttini F: The Victorian ambulatory care sensitive conditions study: rural and urban perspectives. Soz Praventivmed 2003, 48:33-43.

17. Roos LL, Walld R, Uhanova J, Bond R: Physician visits, hospitalizations, and socioeconomic status: ambulatory care sensitive conditions in a Canadian setting. Health Serv Res 2005, 40:1167-85.

18. Stamp KM, Duckett SJ, Fisher DA: Hospital use for potentially preventable conditions in aboriginal and Torres Strait Islander and other Australian populations. Aust N Z J Public Health 1998, 22:673-8.

19. Holman CD, Bass AJ, Rouse IL, Hobbs MS: Population-based linkage of health records in Western Australia: development of a health services research linked database. Aust N Z J Public Health 1999, 23:453-9. 
20. Holman CD, Bass AJ, Rosman DL, Smith MB, Semmens JB, Glasson EJ, et al: A decade of data linkage in Western Australia: strategic design, applications and benefits of the WA data linkage system. Aust Health Rev 2008, 32:766-77.

21. Australian Institute of Health and Welfare: Australian hospital statistics 2008-09. 2010.

22. Lawrence D, Holman CD, Jablensky AV, Threlfall TJ, Fuller SA: Excess cancer mortality in Western Australian psychiatric patients due to higher case fatality rates. Acta Psychiatr Scand 2000, 101:382-8.

23. Australian Bureau of Statistics: Information Paper: Census of Population and Housing - Socio-Economic Indexes for Areas, Australia 2001 Canberra; 2003, Report No:: 2039.0 .

24. Australian Bureau of Statistics: Information Paper: ABS views on remoteness. Canberra; 2001, Report No.: 1244.0.

25. Charlson ME, Pompei P, Ales KL, Mackenzie CR: A new method of classifying prognostic comorbidity in longitudinal studies: development and validation. J Chronic Dis 1987, 40:373-83.

26. Greenland S, Robbins JM: Conceptual problems in the definition and interpretation of attributable fractions. Am J Epidemiol 1988, 128:1185-97.

27. Beyea J, Greenland S: The important of specifying the underlying biologic model in estimating the probability of causation. Health Physics 1999, 76:269-74.

28. Rothman KJ, Greenland S, Lash TL: Modern Epidemiology, Third Edition. Philadelphia: Lippincott; 2008, 62-4.

29. Himelhoch S, Lehman A, Kreyenbuhl J, Daumit G, Brown C, Dixon L: Prevalence of chronic obstructive pulmonary disease among those with serious mental illness. Am J Psychiatry 2004, 161:2317-9.

30. Jones DR, Macias C, Barreira PJ, Fisher WH, Hargreaves WA, Harding CM: Prevalence, severity, and co-occurrence of chronic physical health problems of persons with serious mental illness. Psychiatr Serv 2004 55:1250-7.

31. Starfield B, Shi L, Grover A, Macinko J: The effects of specialist supply on populations' health: assessing the evidence. Health Aff (Millwood) 2005, Suppl Web Exclusives:W5-97-W5-107.

32. Druss BG, Rosenheck RA: Use of medical services by veterans with mental disorders. Psychosomatics 1997, 38:451-8.

\section{Pre-publication history}

The pre-publication history for this paper can be accessed here: http://www.biomedcentral.com/1471-244X/11/163/prepub

\section{Submit your next manuscript to BioMed Central and take full advantage of:}

- Convenient online submission

- Thorough peer review

- No space constraints or color figure charges

- Immediate publication on acceptance

- Inclusion in PubMed, CAS, Scopus and Google Scholar

- Research which is freely available for redistribution

Submit your manuscript at www.biomedcentral.com/submit
Biomed Central 